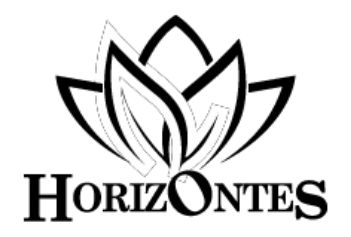

Horizontes. Revista de Investigación en Ciencias de la Educación Https://doi.org/10.33996/revistahorizontes.v2i9.65 enero - marzo 2019 Volumen 3 / No. 9

www.revistahorizontes.org

\title{
Actividad investigativa en el docente universitario
}

\author{
Research activity in the college professor
}

\author{
Wendy C. Montoya G. \\ carolinew_82@hotmail.com
}

Universidad Politécnica Territorial del Norte del Táchira Manuela Sáenz, Venezuela

\section{RESUMEN}

La investigación es la actividad principal de la educación universitaria porque provee a la sociedad de un conocimiento nuevo. La presente investigación tuvo como propósito describir las actividades investigativas de los docentes de la Universidad Politécnica Territorial del Norte del Táchira Manuela Sáenz. Venezuela. La investigación fue de tipo descriptivo con un diseño de campo, transeccional, contemporáneo unieventual. La población la conformaron 70 profesores de la institución estudiada. Para la recolección de los datos se utilizó la técnica de la encuesta con un instrumento escala conformado por 24 ítems, una confiabilidad calculada por Alfa de Cronbach de ,820 lo cual indicó que el instrumento era altamente confiable. En el análisis de los datos se utilizó la estadística descriptiva específicamente la medida de tendencia central mediana por cuanto los datos se midieron en un nivel ordinal. Las conclusiones indicaron que en la institución universitaria la actividad investigativa era regular por lo que se afirmó que los profesores, en su mayoría, no estaban involucrado con la investigaciónpublicación, tampoco con la docenciaasesoría ni con coordinación -evaluación. En tal sentido, no había motivación en el desarrollo de nuevos conocimientos.

Palabras clave: Actividad investigativa; asesoría; coordinación; docencia; investigación

\section{ABSTRACT}

The purpose of this research is to describe the research activities of the teachers of the Northern Tachira Territories Polytechnic University Manuela Sáenz. Venezuela. The research is of a descriptive type with a field design, transectional, contemporary unieventual. The population consists of 70 teachers from the studied institution. To collect the data, the survey technique was used with a scale instrument consisting of 24 items with a reliability calculated by Alpha of Cronbach of 0.82, which indicates that the instrument is highly reliable. In the analysis of the data, descriptive statistics are used specifically the measure of median central tendency as the data was measured at an ordinal level. The conclusions indicate that in the university the investigative activity is regular so it is affirmed that the professors in their majority are not involved with the researchpublication, neither with the teaching-advice nor with coordination-evaluation. Therefore there is no motivation in the development of new knowledge.

Key words: Advice; coordination; research activity; research; teaching 


\section{INTRODUCCIÓN}

El docente universitario tiene como principal labor la actividad investigativa, ya que, a partir de ella surgen los nuevos conocimientos en las diferentes ciencias y disciplinas del saber. La investigación adquiere un papel relevante porque hay una sociedad del conocimiento que es integrada por profesores y estudiantes que hacen de las instituciones recintos donde la sapiencia es el primer medio de producción científico, tecnológico y humanístico para el beneficio de la comunidad globalizada del siglo XXI.

El trabajo científico contribuye a los cambios, transformaciones e innovaciones que ocasionan impacto en las sociedades por sus grandes desafíos en pro de desarrollo humano. La labor del profesor universitario está anclada al progreso de la ciencia desde su área de conocimiento, el aporte que genera a la sociedad es gratificante porque suma beneficios que van de lo interno, o sea, la universidad, a lo externo, la comunidad, el estado, la nación y el mundo.

El presente estudio, se basó en la actividad investigativa del docente universitario y demuestra la importancia que tiene la investigación en los procesos de transformación científica e innovadora.

En las últimas décadas la ciencia, la tecnología, la innovación avanzan exponencialmente y ofrecen al mundo cambios constante para hacer del humano un ser dinámico, dispuesto a estar en consonancia con las novedades que presenta la sociedad del conocimiento y permitir, desde lo científico, el desarrollo social, político, cultural y educativo de un país.

En efecto, la educación universitaria tiene una conexión estrecha con lo social en razón de que las investigaciones que desarrollan las universidades están volcadas a generar soluciones científicas a una sociedad envuelta por el mundo globalizado, como lo manifiesta la Organización de Estados Iberoamericanos, OEI (2004, p. 3) "el conocimiento ha pasado a convertirse en la materia prima fundamental de los procesos productivos contemporáneos".

Por lo tanto, a las universidades que se dedican a investigar y a producir nuevos conocimientos les corresponde continuar en la búsqueda, en la indagación y en la curiosidad de querer saber más para llegar a respuestas que a su vez, generen otras preguntas, es decir, en dichas instituciones este proceso es semejante a una espiral que tiene un inicio, pero no un fin. Hernández (2009) expresa que "la universidad debe promover la apertura de espacios académicos, donde la investigación sea la base de la docencia y la razón de ser de la extensión, proceso educativo que ha de procurar la democratización del saber" (p. 4).

Así, la producción investigativa es fundamental porque constituye uno de los cimientos vitales para la formación del profesional en cualquier área de estudio. Hoy día es necesario realizar investigación, dado al creciente desarrollo científico tecnológico - humanístico que lleva a la vanguardia de los nuevos conocimientos. Al respecto, Bondarenko (2009) manifiesta, "la sociedad del conocimiento plantea la formación de los docentes no para acumular conocimientos sino para investigar, pensar, comunicarse, tener iniciativa, aprender a trabajar por su propia cuenta y de esta manera contribuir al desarrollo comunitario" (p. 3).

Igualmente, el autor antes citado advierte que la investigación tiene como fin construir conocimiento, de nada sirve investigar para una sola persona, se debe difundir de manera que aporte no solo al investigador, sino también a la universidad y a la sociedad que está fuera del espacio académico. 
En tanto, el proceso investigativo origina la interacción y transversación con diferentes disciplinas que, a su vez, logran el desarrollado de innumerables descubrimientos. A partir de la producción investigativa el mundo vive en una construcción constante, de ahí que cada día sale algo nuevo a la palestra. A la investigación se le debe el progreso científico, además del desarrollo de la humanidad.

En relación con lo antes expuesto, las universidades son espacios universales $\mathrm{y}$ están destinadas a producir todo el conocimiento que esté en la capacidad de cada uno de los que en ella hacen vida. Los recintos universitarios permiten traspasar las barreras del saber, y hoy día más cuando la tecnología se cohesiona con la humanidad en todos los aspectos del vivir, de modo que brinda herramientas para llegar a los conocimientos, además de difundirlos tan lejos como la universidad o el investigador lo permita.

En los últimos años la producción científica en Venezuela se encuentra enmarcada por las universidades autónomas, datos arrojados por el Observatorio Nacional de Ciencia, Tecnología e Investigación, ONCTI (2016). Cabe señalar que la educación universitaria está conformada por públicas, privadas, autónomas, politécnicas, experimentales, institutos y colegios universitarios. Asimismo, es de mencionar que, por los problemas económicos en el país, las universidades presentan dificultades en el desarrollo educativo y en los procesos de investigación que se generan en ellas.

Bien lo señala Oppenheimer (2014), al plantear que en la actualidad el progreso de los países está centrado cada vez más en sus sistemas educativos, sus científicos y sus innovadores, en tanto que disminuye la dependencia de sus recursos naturales.

En consideración a lo referido por dicho autor se puede observar que Venezuela es rico en diversos recursos naturales y es un país subdesarrollado. De esta manera en el ámbito educativo venezolano se planean las siguientes interrogantes: ¿en qué punto del aprendizaje académico se enfoca el sistema educativo en todos los niveles de escolaridad?, ¿cuál es la producción de los científicos? ¿A quién beneficia? y ¿qué pasa con los inventos e innovaciones que se desarrollan en la nación?

A tal efecto, Pérez (1996) indica que, "por supuesto debe realizarse investigación en las universidades para la generación de conocimientos aplicados, con la finalidad de que la actividad innovadora se traduzca en beneficio para el aparato productivo". (p. 51) El desafío de realizar investigación está en crear una cultura investigativa y esta labor debe formar parte de la ética de todo profesor universitario.

En la institución objeto de estudio de esta investigación la situación no era diferente porque a pesar de que la universidad contaba con tres grupos de investigación, no había convenios con otras universidades o centros científicos, había escasa producción científica, pocos profesores tenían publicaciones de artículos a nivel nacional e internacional y solo el $14 \%$ de ellos participaban en el Programa Estímulo a la Innovación y a la Investigación PEII, en la categoría A-1; la mayoría de los docentes eran licenciados o ingenieros y no presentaban continuidad en su formación académica.

Igualmente, los docentes no asistían a programas de formación en investigación, ni mostraban interés por el apoyo en asesoría a los estudiantes, al extremo que en algunos casos había quienes se negaban a asesorar las investigaciones que desarrollaban los participantes de la institución.

En función de lo expuesto, surgió el siguiente objetivo de investigación: Describir la actividad investigativa de los docentes de la 
Universidad Politécnica Territorial del Norte del Táchira Manuela Sáenz de La Fría del estado Táchira, para el año 2016.

El estudio de la actividad investigativa en las instituciones de educación superior implica que estas ejerzan la función de investigación, es decir, deben crear estrategias que les permita mantener la gestión del conocimiento. González (2011) dice "la gestión del conocimiento se desarrolla a través de un profesional autónomo y reflexivo con habilidades $\mathrm{y}$ capacidades que son producto de una mezcla de arte, ciencia y oficio" (p. 63).

En tal sentido, la generación de conocimiento se desarrolla mediante el proceso de investigación en el cual el investigador actúa como un gestor del conocimiento porque busca qué investigar, cómo, a dónde dirigir la investigación, organiza sus ideas plasma todos sus pensamientos y los aplica para luego dar a conocer su producto final. Además, el investigador es el gestor en el proceso de investigación que tiene como fin transformar una realidad a través de soluciones científicas.

De esta manera las universidades tienen la responsabilidad de propiciar el proceso de investigación como una actividad propia de su quehacer diario. Al respecto la Unesco (1998), expresa que las instituciones de educación superior tienen el compromiso de:

...promover, generar y difundir conocimientos por medio de la investigación $\mathrm{y}$, como parte de los servicios que ha de prestar a la comunidad, proporcionar las competencias técnicas adecuadas para contribuir al desarrollo cultural, social y económico de las sociedades, fomentando y desarrollando la investigación científica y tecnológica a la par que la investigación en el campo de las ciencias sociales, las humanidades y las artes creativas. (p. 1).
Ante lo cual, se puede afirmar que las universidades son espacios de integración en saberes y un universo de conocimientos que no deben ser enclaustrados. El compromiso de las instituciones universitaria es trabajar con pertinencia para responder desde lo científico a las necesidades sociales.

Vessuri (1996), afirma que, "la profesión académica entendida en términos de profesión científica -esto es, asumiendo las normas y prácticas propias de la producción original de conocimientos- no termina de consolidarse en la mayoría de las universidades". Es conveniente destacar que hoy día existen problemas económicos significativos en muchos países del mundo y Latinoamérica no escapa a ellos, en tal sentido, lo cual hace que la inversión en educación ha sido disminuida lo cual pone en riesgo lo intelectual, científico e innovador y en la calidad de vida de las personas. (p. 32).

$\mathrm{Al}$ respecto, Quintanilla (2008) hace mención que:

...en América Latina y el Caribe se invierte poco. La inversión en investigación y desarrollo está entre 0,1 y $0,6 \%$ del PIB...la inversión anual de toda América Latina y el Caribe es casi la mitad de lo que invierte Corea del Sur (p. 255).

Es necesario que los gobiernos tomen en cuenta que invertir en investigación representa una ganancia para las naciones, bien lo manifestó Fink (s/f c.p. Oppenheimer, (2014), quien en su cargo como jefe economista de la Organización Mundial de la Propiedad Intelectual, (OMPI), expreso "el desafío de los países latinoamericanos es crear un ecosistema donde pueda florecer la innovación...eso implica tener un buen sistema educativo, incentivos fiscales para estimular la investigación y el desarrollo" (p. 29). 
Cabe señalar que países como Argentina, cuenta con el Consejo Nacional de Investigación y la docencia, CONICET, y junto con las universidades nacionales se concentran en crear investigaciones e involucrar a los docentes de las diferentes casas de estudio del país. Según la Agencia de Noticias Bolívar Telám (2012), Argentina es un país que presenta producciones científicas importantes dentro de las que destaca: la modificación de gen de las semillas de girasol, trigo, soja y maíz que permite incrementar significativamente el rendimiento en condiciones de sequía y salinidad, asimismo, la inhibición invitro del proceso neurodegenerativo del Parkinson, clonación de especies amenazadas de extinción, anticuerpos que previene el virus de la fiebre hemorrágica argentina.

Otro país es Chile, en información contentiva en la Comisión Nacional de investigación Científica y Tecnológica, Conicyt (2010) dice que este país "invierte en ciencia y tecnología para obtener nuevo conocimiento, nuevas tecnologías e innovaciones, junto con los beneficios que la ciencia y la tecnología trae consigo en los ámbitos económicos, social, cultural, político, educacional, en salud, ambiental, organizacional y simbólica" (p. 29).

Es así, como Chile investiga sobre los nuevos conocimientos de los antiguos ecosistemas nórticos, los nuevos conocimientos sobre formas cuadráticas, nuevas tecnologías de amortiguaciones antisísmicas, nuevas tecnologías para la identificación de rostros, nuevas tecnologías aéreas para monitorear glaciares, nueva tecnología de exploración teledirigida, nuevas tecnologías para la detección de contaminantes.

Con respecto a Brasil es el país que tiene la mayor producción científica de América latina. Nature (2014) afirma que en este país el estado junto al sector privado realiza una inversión de 27.000 millones de dólares en ciencia, tecnología e innovación. Además, es la única nación en la región que aporta más de $1 \%$ de su economía en el desarrollo del área científica. Asimismo, la Fundación española para la ciencia y la tecnología, FECYT (2017) expresa que en el decenio 2005-2014, Brasil ocupa en el mundo el decimotercer puesto en términos de producción científica.

Cuando se habla de la investigación en Venezuela, es primordial hacer retrospectiva para poder entender cómo se ha desarrollado el proceso investigativo y quienes en la actualidad y quiénes hacen investigación.

Al respecto, Pereira (2007) afirma que para finales del XIX,

En Venezuela la ausencia de una cultura científica autóctona se debió a que la investigación era realizada fundamentalmente por extranjeros para responder a los intereses del dominador, en tanto los blancos criollos personas con formación y capacidad para hacer ciencia se avocaron principalmente a actividades políticas en aras del movimiento independentista (p. 4).

De tal forma que la actividad investigativa se direcciona en un principio únicamente por las universidades, es a finales de los años cuarenta cuando las casas de estudios existentes toman las riendas de la investigación, la primera fue la Universidad Central de Venezuela UCV, luego la Universidad de los Andes ULA, sigue, la Universidad del Zulia LUZ y Universidad Católica Andrés Bello UCAB.

Son las universidades, hasta hoy día, quienes llevan la tarea de crear investigación y de generar conocimientos nuevos, de ahí que cada día existe más compromiso científico dirigido a modificar, transformar o innovar para generar cambios predominantes en la vida del ser humano. La actividad 
investigativa se da a través de sus profesores y de los estudiantes que se enmarcan en un aprendizaje cíclico.

En efecto, la investigación es la causa principal del auge de las universidades, una universidad que no investiga, es una organización que no produce, es estática y no es conocida. Salas (2000) expresa "las universidades constituyen la columna vertebral del subsistema científico-tecnológico, y por eso a ellas les corresponde estimular el espíritu creativo y la investigación científica, que representa el punto de partida de todo esfuerzo de mejoramiento científico-tecnológico" (p. 139).

Dadas las condiciones que anteceden la Ley de Universidades (1970), le da un carácter legal a la actividad de investigación por cuanto en su artículo 3 expresa, "Las Universidades deben realizar una función rectora en la educación, la cultura y la ciencia. Para cumplir esta misión, sus actividades se dirigirán a crear, asimilar y difundir el saber mediante la investigación y la enseñanza".

La actividad investigativa se hace necesaria en la generación de nuevos conocimientos, y son los actores principales como los docentes quienes están llamados a hacer investigación. Narváez y Burgos (2011), afirman que:

La investigación representa una actividad de primer orden en las universidades. Bajo esta óptica, las universidades deberían ser centros de mayor productividad intelectual, si se considera que en el ámbito académico la investigación es un proceso de producción que genera conocimientos (p. 117).

La actividad primordial de las universidades es el hacer investigativo, desde ahí se orienta el aprendizaje, pues la producción investigativa determina la capacidad científica e intelectual del profesorado y de los mismos estudiantes, si no hay investigación, no hay conocimiento nuevo, ni capacidad de inventiva y trae como consecuencia la repetición de temas académicos obsoletos, además del nulo aporte a la sociedad que espera la demostración científica de las instituciones universitarias.

La investigación científica hoy día tiene un mayor auge en comparación a años anteriores, y esto se debe a la densidad poblacional que tiene el país, por ende, las universidades deben generar investigaciones que den solución a problemáticas que puedan ser asistidas a través de la ciencia.

Las universidades venezolanas reciben el respaldo de otras instituciones encargadas de hacer y apoyar la investigación, ya que el Ministerio de Educación Universitaria y el Ministerio de Ciencia y Tecnología se unifican y crean uno que lleva por nombre, Ministerio del Poder Popular para la Educación Universitaria, Ciencia y Tecnología, esto quiere decir que, los centros e institutos de investigación no están separados y se encuentran dentro un solo ministerio.

En este propósito, es conveniente mencionar las instituciones que brindan conocimiento científico y en su mayoría tienen convenios con universidades de la nación, dentro de ellos están: el Instituto Venezolano de Investigación Científica (IVIC), el Fondo Nacional de Ciencia y Tecnología (FONACIT), el Centro de Investigaciones del Estado para la Producción Experimental Agroindustrial (CIEPE), el Centro de Investigaciones de Astronomía "Francisco J. Duarte" (CIDA) y la Fundación Venezolana de Investigaciones Sismológicas (FUNVISIS).

Además, se encuentra el Observatorio Nacional de Ciencia, Tecnología e Innovación (ONCTI) cuyo objetivo se orienta a proveer la información aplicada a diversas áreas del Ministerio del poder popular de Ciencia, Tecnología e Innovación, para satisfacer la 
demanda que surge de las instituciones públicas $\mathrm{y}$ del poder popular, a fin de promover y fortalecer el desarrollo científico y tecnológico, con impacto en lo político, productivo, ambiental y social, que contribuya con la soberanía nacional.

En la ONCTI se encuentra el Programa Estímulo a la Innovación y la Investigación PEII que a través del Registro Nacional de Innovadores e investigadores RNII, el innovador o investigador se registra y sigue una serie de pasos y requisitos que pasan por evaluadores quienes determinarán si cumple con los requerimientos y lo ubicarán en el nivel A1- A2, B y C que conforman el PEII.

Según estadísticas de Morales, Medina y Álvarez (2003), para el año 2000 la situación de la investigación científica en Venezuela fue la siguiente: más del $70 \%$ de dicha actividad se llevó a cabo en las instituciones de educación superior, sobre todo en las universidades. Orientaciones dadas por las estadísticas del Programa de Promoción al Investigador, un $25 \%$ se realizó en la UCV, $16 \%$ en la ULA y $12 \%$ en la USB y el IVIC con un total de $11 \%$ de investigadores.

En tanto el Observatorio Nacional de Ciencia, Tecnología e Innovación (ONCTI) emitió un registro desde el año 2011 hasta el año 2015 donde se aprecia que los innovadores e investigadores independientes tienen 43.459 productos de investigación acreditados, le siguen las universidades autónomas con 17.285, las experimentales presentan 10.953, los institutos y colegios privados, 620 y son los que menos producción investigativa registran.

De tal manera que, son los innovadores e investigadores independientes los que más producción científica hacen, por tanto, es importante el número de personas que no pertenecen a alguna institución y aportan nuevos conocimientos a la sociedad.
Asimismo, ONCTI en sus estadísticas da a conocer los productos de investigación acreditados al programa PEII, desde el año 2011 al 2015, los artículos de investigación publicados en revistas arbitradas tienen en total 30.152 publicaciones en actas arbitradas de memorias y conferencias; 20.252 trabajos de maestría, tesis de doctorado o trabajos de ascenso; 10.758 libros y capítulos en libros; 9.878 productos de proyectos de investigación, acción participativa; 2949 sistematización de experiencias; 685 patentes de invención, mejoras y modelos, más 72 dibujos industriales.

Puede notarse que la mayor cantidad de productos de investigación lo representan los artículos científicos, por lo que es importante reconocer la labor del investigador en dar a conocer sus producciones, de esta manera da auge al trabajo investigativo que se realiza en el país y contribuye en la globalización del conocimiento. De igual manera puede apreciarse el bajo número en patentes de invención.

Entre tanto, ONCTI describe las áreas del conocimiento que más publicaciones tienen desde el año 2011 al 2015, dentro de ellas se encuentran como primera área, ciencias naturales y exactas con 4382 publicaciones, le sigue, ciencias agrícolas con 3595 , humanidades con 3589, ingeniaría y tecnología tienen publicadas 3220 producciones, ciencias sociales aparece con 3504 y por último las ciencias médicas con un total de 2142 publicaciones.

En este sentido, es necesario que las universidades e instituciones de investigación se comprometan más con la producción investigativa, aunque Venezuela está en constante cambio y con problemas económicos, esto hace que los investigadores y científicos deben contribuir a la mejora del país a través de la ciencia, pues es un desafío que consiste en acrecentar la educación que es vital para el crecimiento y desarrollo de la nación. 
Por lo tanto, es necesaria la formación del profesorado en la actividad científica ya que una universidad que no produzca investigación es una universidad vacía en pensamientos propios. En tal medida, la producción científica de una universidad va a depender de su cultura investigativa.

Restrepo (2009, c.p. Aparicio 2008), expresa que:

La cultura investigativa es aquella que comprende, toda manifestación cultural, organizaciones, actitudes, valores, objetos, métodos y técnicas, todo en relación con la investigación, así como la transmisión de la investigación o pedagogía de la misma. La cultura no se inicia con sistemas, pero los va conformando. La cultura de la investigación es iniciada por profesores individuales, pero poco a poco integra equipos, grupos, comités y centros de investigación y desarrollo tecnológico $\mathrm{y}$ redes que tejen el sistema de investigación en la universidad. (p. 3).

Por consiguiente, el profesor universitario tiene el deber de integrarse en todo aquello que esté relacionado con la actividad investigativa, el deber ser es trabajar bajo una línea de investigación de acuerdo a su perfil académico, donde se sienta en capacidad de generar aportes tanto individuales como en grupos de investigación.

En tanto que, si se forja la cultura de la investigación a través de la formación permanente del docente universitario, éste comprenderá que el fin de trabajar en la educación universitaria radica en aportar conocimiento nuevo a los estudiantes y a la sociedad en general.

En efecto, el docente es la base fundamental del proceso educativo, pues es quien transmite los conocimientos y estos serán multiplicados y puestos en prácticas en diferentes áreas de estudios. De ahí la importancia de la capacitación constante del docente universitario para que este en consonancia de las novedades que surgen en el mundo. Así, Narváez y Burgos (2011), anuncian que:

La docencia y la investigación junto a las actividades de extensión representan los componentes del trabajo académico que debe desempeñar todo profesor universitario con miras a elevar la calidad de su formación integral si se considera como procesos complementarios y articulados. (p. 117).

De manera que, la investigación es la principal labor del docente universitario, desde este punto el docente se convierte en un profesional crítico-reflexivo en un área específica, bajo una línea de investigación, lo que permite estar actualizado en los nuevos acontecimientos, nuevos avances conceptuales y tecnológicas, nuevos métodos de aprendizajes. El docente universitario, es un ser comprometido con el acontecer del conocimiento.

$\mathrm{Si}$ el docente se forma, se instruye constantemente, es un hecho que los estudiantes van a adquirir conocimientos novedosos que conducen a la productividad dentro y fuera de los espacios universitarios, el estudiante se direcciona hacía nuevos enfoques, hacia metodologías para abordar una investigación, de tal manera la universidad se convierte en generadora de productos científicos creados no solo por el docente, sino también por los estudiantes.

Pero, la actividad investigativa en la formación del docente se da a partir de la gestión de los entes rectores dentro de los espacios universitarios y de la misma iniciativa del docente por querer superarse cada día. Según Restrepo (2009, p. 106), se debe "replantear los modelos de formación de formadores, que buscan posicionar la figura 
de un maestro investigador capaz de alentar la investigación, la participación y la innovación".

El docente que no investiga y no tiene la formación investigativa, al pasar el tiempo sus conocimientos se convierten en obsoletos, y el estudiante de hoy día no es un ser pasivo, es una persona que vive informada de una u otra forma a través de la invasión de medios de comunicación, específicamente los medios cibernéticos, que han hecho que la información esté a la mano a cualquier hora del día, lo que permite al mismo estudiante verificar si lo que dice un profesor es verdad o mentira o si está actualizado en sus conocimientos.

La formación del docente es importante porque esta lo instruye para el mundo competitivo donde se encuentra, lo aparta de lo común y lo induce a otras formas de pensar, de concebir el conocimiento, de formar y tener su propio paradigma ante las cosas, conocer que para investigar no solo existe un método, un tipo de investigación; a diferenciar entre tópicos que resultan confusos lo cual evita que en ocasiones sus contrariedades las transmita a los estudiantes quienes luego las convierten en vicios.

Es por esas razones que, resulta importante la asesoría en el proceso investigativo, dicha actividad es guiada por personas expertas en el área que fomentan el desarrollo de competencias de investigación y el interés por la ciencia a través de la formación, el seguimiento y la dirección de la investigación.

En este sentido, no solo la inducción hacia la investigación es suficiente, sino también, la orientación de que se realice bajo la metodología adecuada, pues muchas investigaciones comienzan, pero se quedan en el abandono y por falta de seguimiento $y$ evaluación se desperdicia un conocimiento que probablemente es una producción científica generadora de nuevos aportes.

En referencia con lo antes expuesto, es necesario que las coordinaciones o departamentos de investigación tomen como prioridad la actividad de asesoría en el docente, de esta manera se crea motivación, gusto, interés hacia la actividad investigativa. Además, se debe tomar en cuenta que en la coordinación y evaluación existe un liderazgo que orienta a promover nuevos conocimientos en la institución, a través de iniciativas, seguimiento, evaluación y reorientación de los proyectos de investigación.

Es así que, a partir de la formación del docente, éste puede transformar aspectos cotidianos y convertirlos en conocimientos científicos. Como expresa Arteaga (2009), el docente investigador tiene la autoridad que le confiere el ser constructor y creador de conocimiento a partir de sus propias investigaciones, a diferencia del docente repetidor de teorías ajenas.

Pues bien, Aldana (2012), menciona algunos aspectos a tener en cuenta para fortalecer la formación investigativa del docente: primero, el docente como enseñante de investigación; debe estar preparado para formar seres humanos disciplinados, perseverantes, curiosos frente a la ciencia, la tecnología y la humanidad. Segundo, el docente universitario debe tomar en serio la tarea de docente investigador, pues de él depende que tan productiva sea la estadía del estudiante dentro de la universidad.

Seguidamente, como tercer aspecto, las competencias básicas para la investigación; el docente universitario debe tener competencia, habilidades, destrezas que lo definan en el ambiente universitario, si no tiene la formación investigativa puede construir aprendizajes en investigación para una pertinente labor universitaria.

Igualmente, en el cuarto punto se encuentra el compromiso institucional frente 
a la investigación; el docente debe estar consciente de la responsabilidad que tiene al estar frente a las actividades académicas universitarias, con el compromiso en la investigación y la formación de conocimientos; asimismo debe saber que el mundo necesita personas productivas que quieran aportar ideas, pensamientos que nutran las debilidades presentes en la sociedad.

En tal sentido, el docente debe tener la iniciativa de mejorar cada día su forma de actuar dentro de los espacios académicos, el compromiso con su formación pedagógica, su producción investigativa y a su vez la difusión del conocimiento. En la medida que el docente se prepara y produce, éste crece como persona, como profesional, y a su vez, progresa la universidad con conocimiento científico que beneficia no solo lo interno del espacio académico, sino también, todo lo externo que esté en correspondencia con el espacio universitario.

\section{MÉTODO}

En este caso, se basó en describir la actividad investigativa de los docentes de la UPTNT Manuela Sáenz. El diseño de la investigación se enmarcó según el origen de los datos en un diseño de campo, de acuerdo a la perspectiva de temporalidad, el estudio fue transeccional contemporáneo porque los datos se recolectaron en un solo momento. Con respecto a la amplitud del foco es univariable.

La población la conformaron 70 docentes que laboraban en la universidad estudiada, en los diferentes programas nacionales de formación y programa carrera que imparte dicha casa de estudio.

Para la recolección de los datos se utilizó la técnica de la encuesta con un instrumento cuestionario contentivo de 24 ítems. La confiabilidad se calculó por Alfa de Cronbach mediante el programa SPSS 18 lo cual arrojó un valor de 0,82 que indicó que el instrumento es altamente confiable.

Para la investigación se definió la actividad investigativa como las actividades de investigación y publicación, docencia y asesoría y coordinación y evaluación que realizan los docentes en el espacio universitario. Las técnicas de análisis utilizadas fueron las relacionadas con la estadística descriptiva, específicamente la medida de tendencia central, la mediana y las frecuencias absolutas y porcentuales.

El análisis se interpretó mediante el baremo de interpretación que se presenta en la tabla 1.

Tabla 1. Baremo de interpretación

\begin{tabular}{cc}
\hline Categoría & Interpretación \\
\hline 0 a 3,99 & Actividad muy deficiente \\
4 a 7,99 & Actividad deficiente \\
8 a 11,99 & Actividad regular \\
12 a 14,99 & Actividad suficiente \\
15 a 20 & Actividad excelente \\
\hline
\end{tabular}


RESULTADOS

Con relación a la actividad investigativa los resultados que se presentan en la tabla 2 muestran una mediana de 5,6 puntos de un máximo de 20 puntos que al interpretarse con el baremo indica una actividad investigativa deficiente, lo cual refleja que los docentes de la institución no desarrollaban publicaciones, ni trabajaban con procesos de asesoría y asistencia a eventos.

Tabla 2. Estadísticos de actividad investigativa

\begin{tabular}{lll}
\hline $\mathbf{N}$ & $\begin{array}{l}\text { Valid } \\
\text { Missing }\end{array}$ & $\begin{array}{l}\mathbf{7 0} \\
\mathbf{0}\end{array}$ \\
\hline Mediana & & 5,6 \\
Mínimo & &, 0 \\
Máximo & & 16,0 \\
Percentiles & 25 & 3,2 \\
& 50 & 5,6 \\
& 75 & 8,8 \\
\hline
\end{tabular}

Con respecto a cómo se distribuyen los docenes en las categorías de actividad investigativa se observa en la tabla 3 que un $34,3 \%$ se ubicó en las categorías de deficiente y regular respectivamente, un $27,1 \%$ en muy deficiente, un $2.9 \%$ en suficiente y solo un $1,4 \%$ en excelente actividad investigativa

Tabla 3. Categorías de Actividad Investigativa

\begin{tabular}{|c|c|c|c|c|c|}
\hline & & Frecuencia & Porcentaje & $\begin{array}{c}\text { Porcentaje } \\
\text { válido }\end{array}$ & $\begin{array}{l}\text { Porcentaje } \\
\text { acumulado } \\
\end{array}$ \\
\hline \multirow[t]{6}{*}{ Valid } & $\begin{array}{l}\text { Actividad muy } \\
\text { deficiente }\end{array}$ & 19 & 27,1 & 27,1 & 27,1 \\
\hline & Actividad deficiente & 24 & 34,3 & 34,3 & 61,4 \\
\hline & Actividad regular & 24 & 34,3 & 34,3 & 95,7 \\
\hline & Actividad suficiente & 2 & 2,9 & 2,9 & 98,6 \\
\hline & Actividad excelente & 1 & 1,4 & 1,4 & 100,0 \\
\hline & Total & 70 & 100,0 & 100,0 & \\
\hline
\end{tabular}

\section{Sinergias de actividad investigativa}

Con respecto a las sinergias del evento actividad investigativa se observa en la tabla 4 que todas las sinergias se ubicaron, según el baremo de interpretación, en deficiente (investigación y producción 6,6 puntos, docencia y asesoría 7,50 puntos y coordinación evaluación 5,00 puntos). Ante lo cual se puede afirmar que en la institución estudiada los docentes no realizaban actividades dirigidas a la investigación. 
Tabla 4. Estadísticos de las sinergias de actividad investigativa

\begin{tabular}{|c|c|c|c|c|}
\hline & & $\begin{array}{c}\text { Puntaje } \\
\text { transformado } \\
\text { investigación y } \\
\text { producción }\end{array}$ & $\begin{array}{c}\text { Puntaje } \\
\text { transformado } \\
\text { docencia y } \\
\text { asesoría }\end{array}$ & $\begin{array}{c}\text { Puntaje } \\
\text { transformado } \\
\text { coordinación y } \\
\text { evaluación }\end{array}$ \\
\hline \multirow[t]{2}{*}{$\mathrm{N}$} & Valid & 70 & 70 & 70 \\
\hline & Missing & 0 & 0 & 0 \\
\hline Mediana & & 6,6 & 7,5 & 5,0 \\
\hline Mínimo & & 0 & 0 & 0 \\
\hline Máximo & & 17,8 & 18 & 13 \\
\hline \multirow[t]{3}{*}{ Percentiles } & 25 & 2,2 & 2,5 & 00 \\
\hline & 50 & 6,6 & 7,5 & 5,0 \\
\hline & 75 & 11,1 & 12,5 & 7,5 \\
\hline
\end{tabular}

Con respecto a la distribución de la población en las categorías de investigación y producción se observa en la tabla 5 que un $62,8 \%$ de la población desarrollaba investigaciones y publicaciones de forma deficiente y muy deficiente, un $22,9 \%$ lo desarrollaba en forma regular y solo un $14 \%$ desarrollaba esas actividades de manera suficiente.

Tabla 5. Categorías de investigación y publicación

\begin{tabular}{|c|c|c|c|c|c|}
\hline & & Frecuencia & Porcentaje & $\begin{array}{c}\text { Porcentaje } \\
\text { válido }\end{array}$ & $\begin{array}{l}\text { Porcentaje } \\
\text { Acumulado }\end{array}$ \\
\hline \multirow[t]{6}{*}{ Valid } & $\begin{array}{l}\text { Actividad muy } \\
\text { deficiente }\end{array}$ & 22 & 31,4 & 31,4 & 31,4 \\
\hline & Actividad deficiente & 22 & 31,4 & 31,4 & 62,9 \\
\hline & Actividad regular & 16 & 22,9 & 22,9 & 85,7 \\
\hline & Actividad suficiente & 7 & 10,0 & 10,0 & 95,7 \\
\hline & Actividad excelente & 3 & 4,3 & 4,3 & 100,0 \\
\hline & Total & 70 & 100,0 & 100,0 & \\
\hline
\end{tabular}

En el gráfico 1 se presentan los resultados de los ítems de la sinergia investigación y publicación, donde se observa que las actividades con mayores deficiencias en el cumplimiento por parte de los docentes eran las referidas a pertenecer a un equipo de investigación (50\%), la publicación de ponencias y artículos (63\%) y la presentación de ponencias en eventos científicos $(62 \%)$ 


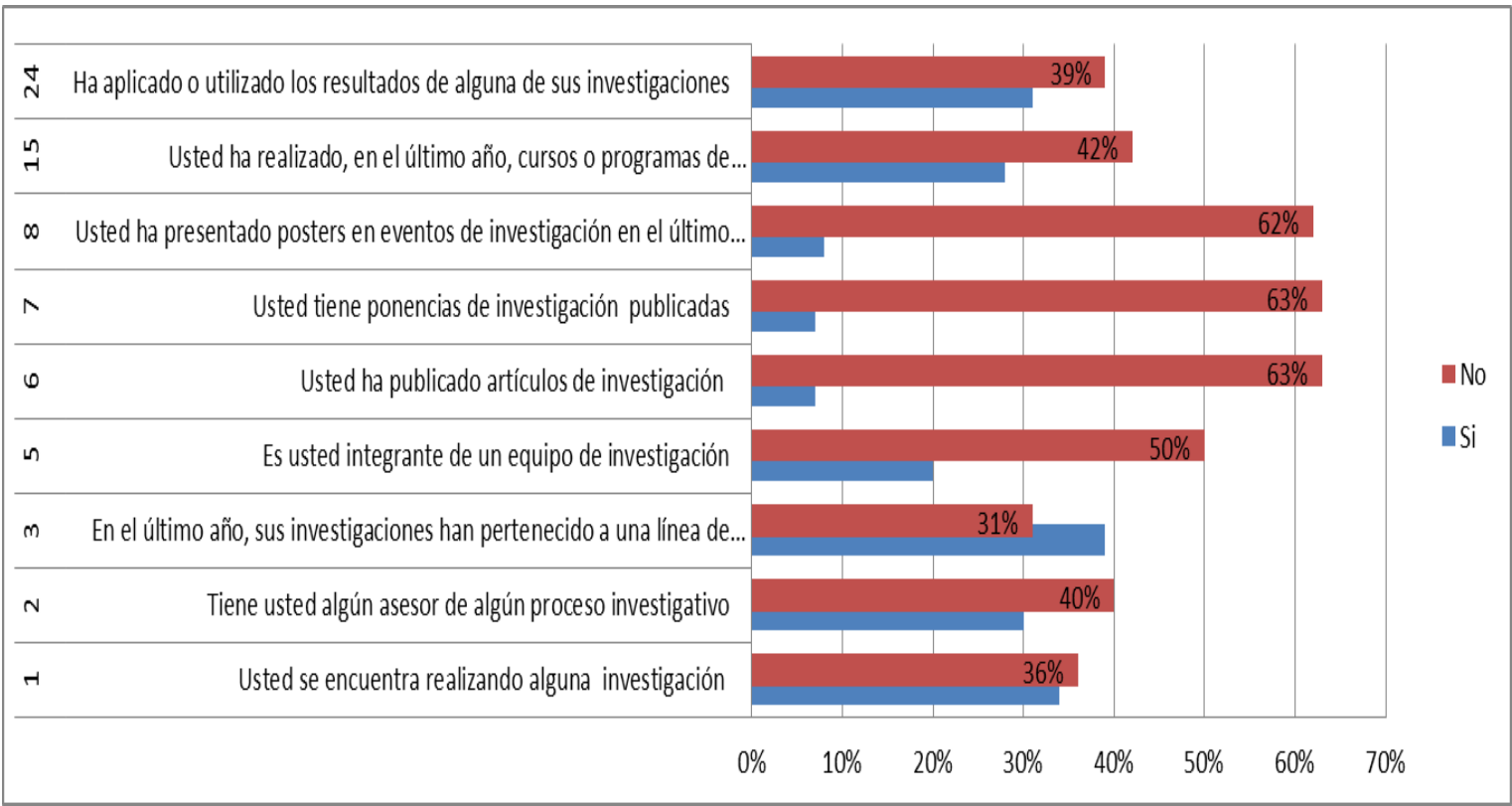

Gráfico 1. Representación porcentual de los ítems de la sinergia investigación-publicación

Los resultados de la distribución de los docentes en las categorías de la sinergia docencia y asesoría se presentaron en la tabla 6 , donde se observa que la mayoría de los docentes se ubicó en la categoría de muy deficiente (32\%) y deficiente $(25,7 \%)$, lo cual indicaba que éstos no incorporaban en sus clases los resultados de las investigaciones ni realizaban actividades docentes referidas al proceso de investigación. Un 41,2\% se ubicaba en las categorías de regular, suficiente y excelente.

Tabla 6. Categorías de Docencia y Asesoría

\begin{tabular}{|c|c|c|c|c|c|}
\hline & & Frecuencia & Porcentaje & $\begin{array}{l}\text { Porcentaje } \\
\text { válido }\end{array}$ & $\begin{array}{l}\text { Porcentaje } \\
\text { Acumulado }\end{array}$ \\
\hline \multirow[t]{6}{*}{ Valid } & $\begin{array}{l}\text { Actividad muy } \\
\text { deficiente }\end{array}$ & 23 & 32,9 & 32,9 & 32,9 \\
\hline & Actividad deficiente & 18 & 25,7 & 25,7 & 58,6 \\
\hline & Actividad regular & 9 & 12,9 & 12,9 & 71,4 \\
\hline & Actividad suficiente & 17 & 24,3 & 24,3 & 95,7 \\
\hline & Actividad excelente & 3 & 4,3 & 4,3 & 100,0 \\
\hline & Total & 70 & 100,0 & 100,0 & \\
\hline
\end{tabular}




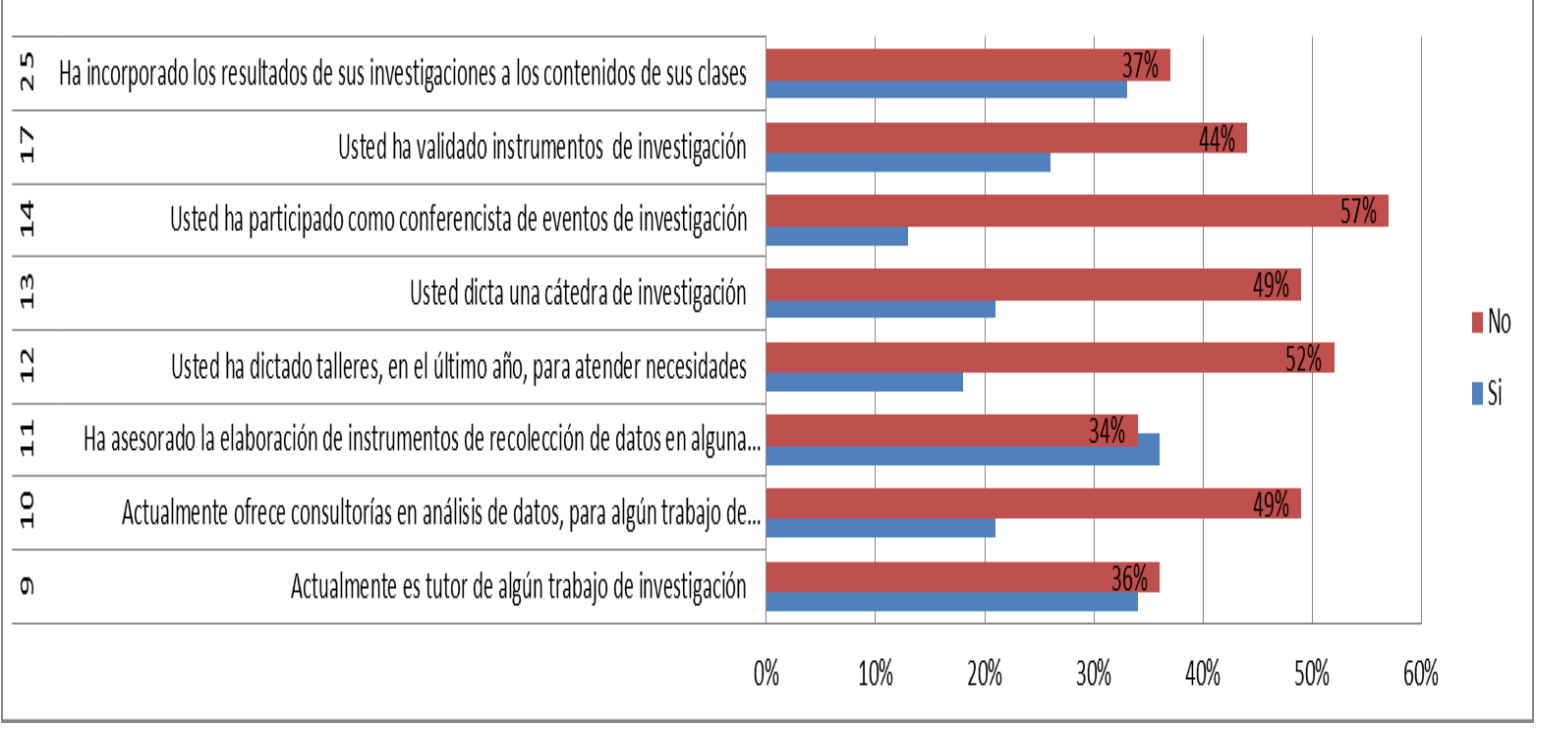

Gráfico 2. Porcentaje de la sinergia docencia-asesoría.

En el gráfico 2, se puede observar que las actividades que los docentes menos realizaban eran las de consultoría en análisis de datos para la investigación 49\%, dictar talleres para atender necesidades académicas $52 \%$, dictar clases en asignaturas de investigación $49 \%$ y participar como conferencista en eventos científicos $57 \%$.

La tabla 7 muestra que un $94,3 \%$ de los profesores desarrollaban una coordinación y evaluación en investigación deficiente y muy deficiente y solo un 5,7\% se ubicó en la categoría de regular y suficiente, lo cual indica que no existe una gerencia o un liderazgo dentro de la universidad que incentive a los profesores a trabajar en las actividades referidas a gerenciar la investigación en la institución.

Tabla 7. Categorías de coordinación y evaluación

\begin{tabular}{llrrrr}
\hline & Frecuencia & Porcentaje & $\begin{array}{c}\text { Porcentaje } \\
\text { válido }\end{array}$ & $\begin{array}{c}\text { Porcentaje } \\
\text { Acumulado }\end{array}$ \\
\hline Valid & Actividad muy & 28 & 40,0 & 40,0 & 40,0 \\
& deficiente & & & & \\
& Actividad deficiente & 38 & 54,3 & 54,3 & 94,3 \\
& Actividad regular & 3 & 1,4 & 1,4 & 95,7 \\
& Actividad suficiente & 4,3 & 4,3 & 100,0 \\
\cline { 2 - 6 } & Total & 70 & 100,0 & 100,0 & \\
\hline
\end{tabular}




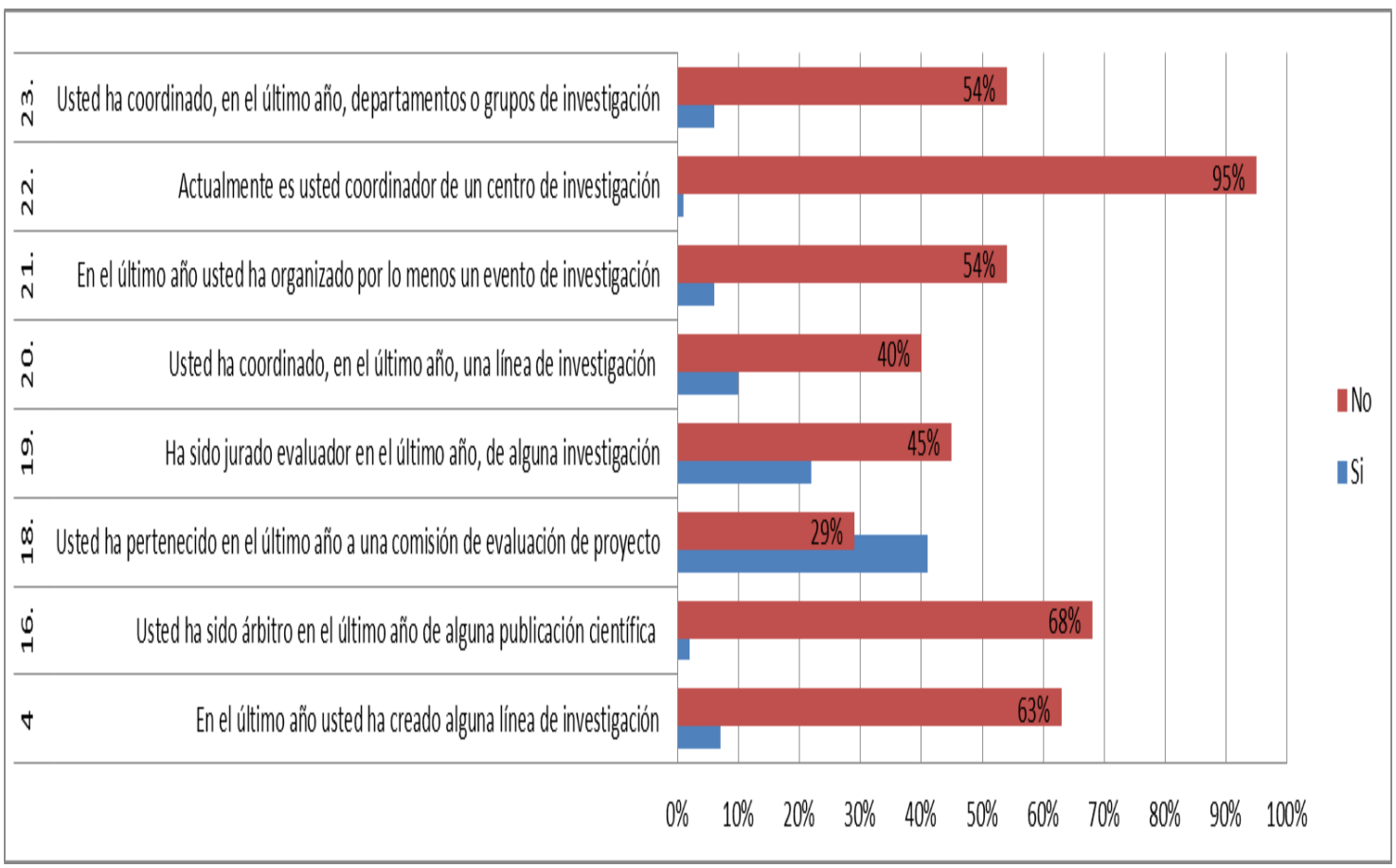

Gráfico 3. Porcentaje de la sinergia coordinación y evaluación

El gráfico 3 muestra que las actividades de investigación menos realizadas por los docentes de la institución estudiada eran la coordinación de los centros de investigación 95\%, creación de líneas de investigación 63\%, ser árbitro en una revista científica 68\%, coordinar departamentos de investigación $54 \%$ y organizar eventos de investigación $54 \%$.

\section{Discusión}

Los resultados demuestran que en la institución estudiada la actividad investigativa que desarrollaban los docentes era deficiente lo cual reflejaba que la investigación no era prioridad en los procesos académicos de esa institución.

Estos resultados contradicen la esencia de las instituciones universitarias expresadas por la Unesco (1998) cuando plantea que las universidades tienen el deber de promover, generar y difundir el conocimiento mediante la investigación.

La actividad investigativa va más allá de hacer investigación, esto quiere decir que el docente investigador se comporta como un gerente líder, donde también coordina grupos de investigación, evalúa las actividades investigativas de los demás profesores, los motiva y gestiona para que asistan a eventos académicos, esto con el fin de integrarlos a una sociedad científica que actúa exponencialmente con el fin de aportar soluciones desde lo científico a problemas que el individuo enfrenta constantemente

En cuanto a la investigación-publicación se puede afirmar, según los resultados con una mediana ubicada en la categoría de deficiente, que en la institución esta sinergia no era un factor predominante, la mayoría de los profesores no realizaban producción científica y por tal motivo no publicaban, asimismo, no participaban como ponentes, entre otras actividades que se logran con la actividad investigativa.

A este respecto, es deber de los actores de la universidad impulsar la investigación dentro de la academia, pues esta actividad es base fundamental a nivel universitario, las universidades están llamadas a producir ciencia, a desarrollar nuevas ideas y a innovar en pro del crecimiento institucional, profesional y del país. No involucrarse con la investigación, corre el riesgo a impartir un 
conocimiento que ya caducó, además de quedar atrasado a todos los acontecimientos que se generan en el mundo globalizado

Las universidades de hoy se involucran más con las comunidades, dan soluciones desde lo académico, investigativo y científico a los problemas que se presentan, ya no están enclaustradas en un salón de clases, el conocimiento que se produce lo comparten y se aplican. Por lo tanto, no debe existir una universidad estática, es necesario crear planes y programas que motiven y fomenten la cultura investigativa en los profesores para que sean profesionales activos que produzcan conocimientos, que se formen cada día y obtengan productividad intelectual con el propósito de beneficiar a la sociedad.

Los profesores que investigan y publican, nutren de información novedosa a los estudiantes, sin embargo, los resultados referidos a la docencia y asesoría ubicados en una categoría de deficiente indicaron que los profesores de la universidad estudiada no desarrollaban procesos de asesoría e incorporación de los resultados de sus investigaciones en sus clases, lo cual evita que el profesor mantenga actualizada su asignatura.

Además, la falta de participación en los eventos científicos a nivel nacional e internacional, disminuye la oportunidad de que el docente se mantenga en contactos con investigadores de otras instituciones a fin de lograr crear enlaces, convenios que beneficien a la universidad donde pertenece. La universidad debe tener responsabilidad social y no estar ajena a lo que acontece, el deber es aportar con conocimiento.

En razón de lo anteriormente planteado puede explicarse los resultados negativos obtenidos por la institución universitaria, ante lo cual es necesario que se desarrollen estrategias en beneficio de una producción científica activa, participativa e integrativa que converja con las necesidades de la población objeto de estudio.

Por lo tanto, si no hay investigación el profesor se ve limitado solo a impartir un conocimiento en un área específica, este hecho limita la oportunidad de abrirse a un campo donde las oportunidades están dadas para crecer en lo intelectual. De manera que, el docente universitario que no se integra a la actividad investigativa, nunca representará a la institución en eventos académicos y trae como consecuencia la desvinculación con el mundo científico y el nulo reconocimiento como institución universitaria.

En tal sentido, el docente no debe estar divorciado del hacer científico porque esta tarea determina la responsabilidad de la universidad hacia la sociedad y productividad calificada en el país y a nivel mundial. En una concepción más amplia, el compromiso que tienen los profesores por realizar investigación es el grado de calidad institucional y académica que refleja la casa de estudio universitaria.

Es determinante apreciar que la labor investigativa no tiene gran importancia en el quehacer universitario, los docentes de dicha universidad no se integran a la actividad científica dentro y fuera de la institución académica y además no interactúan con otras casas de estudios en cuanto a las novedades científicas y a la producción innovadora que genera el mundo académico.

Es deber que un profesor universitario integre un grupo de investigación, de esta manera se enfoca en una línea que lo determina y lo conduce a producir ciencia en un área específica que abarque todas sus dimensiones. A partir de la actividad investigativa se desprenden otras actividades, como la asesoría, factor significativo pues permite que los proyectos de investigación guarden pertinencia con las realidades que se presentan en la sociedad, así también, motiva e impulsa a generar conocimientos nuevos. Por tanto, investigar hace que las universidades se cohesionen y se centren en crear producción desde lo científico, tecnológico y humanístico.

Las universidades deben apostar a los cambios, a la dinámica de ir en consonancia a la realidad de la sociedad y a las transformaciones que se gestan en el transcurrir del tiempo, una casa de estudio que solo se dedique a impartir conocimientos y no a producir, es una institución que se sumerge en el abandono académico, y se 
convierte en un problema social porque las universidades están para brindar soluciones y conocimientos desde sus propias investigaciones, se encargan de sumar y no de restar, ellas están facultadas para convertir al estudiante en talentos para la prosperidad social, económica, educativa del país, no de repetir un conocimiento que ya es obsoleto y no contribuye a una nación que tiene carencias y ansías de progresar.

\section{CONCLUSIONES}

Con respecto a la actividad investigativa de los profesores de la Universidad Politécnica Territorial del Norte del Táchira Manuela Sáenz los resultados indicaron que era deficiente lo cual puso en evidencia que la investigación no era prioridad en los procesos académicos de esa institución. No involucrarse con la investigación es correr el riesgo de impartir un conocimiento que ya caducó, además de quedar atrasados a todos los acontecimientos que se generan en el mundo globalizado. Los docentes, en conjunto con las universidades, están llamados a producir ciencia, a desarrollar nuevas ideas y a innovar en pro del crecimiento institucional, profesional y del país.

Con relación a la investigaciónpublicación ubicada en la categoría de deficiente se puede afirmar que en la institución esta sinergia no era un factor predominante, en su mayoría los profesores no realizaban producción científica y por tal motivo no publicaban, asimismo, no participaban como ponentes, entre otras actividades que se logran con la actividad investigativa. Ante esto puede inferirse que los profesores no están involucrados con la actividad investigativa, solo se dedican a impartir clases a los estudiantes de pregrado.

Los resultados de la sinergia docenciaasesoría ubicada en una categoría de deficiente indicó que los profesores de la universidad estudiada no desarrollaban procesos de asesoría e incorporación de los resultados de sus investigaciones en sus clases, lo cual evitaba que el profesor mantuviese actualizada su asignatura
Además, se identificó que algunos profesores asesoraban trabajos de investigación cuando les correspondía la unidad curricular proyecto tutorial o cuando eran seleccionados como jurados en las defensas de los proyectos de los estudiantes. Esta situación permite afirmar que los docentes solo imparten los conocimientos en su área específica.

De igual manera, se puede observar que los resultados de la sinergia coordinación y evaluación se ubicaron en la categoría deficiente y muy deficiente, lo cual indicaba que no existía una gerencia o un liderazgo dentro de la universidad, por lo tanto, los profesores no participaban en las líneas de investigación de la institución y no tenían incentivos para coordinar las áreas de investigación.

\section{REFERENCIAS}

Aldana, G. (2012). Formación investigativa: su pertinencia en pregrado. Revista Virtual Universidad Católica del Norte. $\mathrm{N}^{\circ} 35$ febrero-mayo

Aparicio, X. (2008). La investigación formativa y el líder servidor en la educación superior. CONHIEREMI, revista universitaria de investigación y diálogo académico. Volumen $4 \mathrm{~N}^{\circ} 1$

Arteaga, I. (2009). El docente investigador en la formación de profesionales. Revista Virtual Universidad Católica del Norte. Nro. 27 mayo-junio

Bondarenko, N. (2009). El componente investigativo y la formación docente en Venezuela. Revista virtual Estudios Pedagógicos. Vol. XXXV, núm. 1. Consultado el 20 de junio de 2017 en: http://www.redalyc.org/pdf/1735/173514 138015.pdf

Comisión Nacional de Investigación Científica y Tecnológica. Conicyt. (2010). Ciencia y Tecnología en Chile ¿Para qué? Verde identidad y comunicación: Santiago Chile

Fundación española para la ciencia y la tecnología, FECYT. (2017). https://icono.fecyt.es/sites/default/files/fil epublicaciones/produccion_cientifica_espan a_y_brasil.pdf 
González, C. (2011). Las reformas estandarizadoras y la gestión del conocimiento pedagógico. Una aproximación desde las representaciones sociales de los profesores. Revista de estudios y experiencias en educación. Vol. 10, núm. 20 agosto-diciembre. Consultada el 20 de junio de 2017 en: http://www.redalyc.org/articulo.oa?id=243 122668003

Hernández, I. (2009). El docente investigador en la formación de profesionales. Revista virtual Universidad Católica del Norte. Nro. 27 mayo-agosto.

Ley de universidades. (1970). Gaceta Oficial $\mathrm{N}^{\circ}$ 1429, Extraordinario del 08-09-70.

Morales, V, Medina, E y Álvarez N. (2003). La educación superior en Venezuela.

Narváez y Burgos (2011) La productividad investigativa del docente universitario. Venezuela: Revista científica electrónica de ciencias y humanidades.

Nature (2014). La ciencia en Sudamérica. https://www.todojujuy.com/mundo/laciencia-sudamerica-n22481

Observatorio Nacional de Ciencia, Tecnología e Innovación ONCTI. https://www.mppeuct.gob.ve/ministerio/di rectorio/entes-adscritos/oncti

Oppenheimer, A. (2014). ¡Crear o Morir! La esperanza de Latinoamérica y las cinco claves de la innovación. Nueva York: Debate.

Organización de Estado Iberoamericanos. OEI (2004). Globalización, ciencia y tecnología. https://www.oei.es/historico/oeivirt/tema svol2.pdf

Pereira, L. (2007). Principales obstáculos que inciden en el desarrollo de la investigación. XXII congreso de la Asociación Latinoamericana de Sociología. Asociación Latinoamericana de Sociología de Guadalajara.

Pérez, J. (1996). Las universidades y la investigación científica en Venezuela. Instituto oceanográfico de Venezuela. Universidad de Oriente. Cumaná-Venezuela.

Quintanilla, A. (2008). La producción de conocimiento en América Latina. Revista Salud Colectiva.4 (3) septiembre-diciembre: Buenos Aires, Argentina.

Restrepo, B. (2009). Investigación de Aula: formas y actores. Revista Educación y Pedagogía. Medellín, Universidad de Antioquia, Facultad de Educación, Vol. 21, número 53 , enero-abril

Salas, R. (2000). La calidad en el desarrollo profesional. Avances y desafíos. [Página web en línea]. Consultado el 10 de julio de 2017 en:

http://scielo.sld.cu/scielo.php?script=sci_art text\&pid=S0864-21412000000200003

Telám

http://www.telam.com.ar/notas/201212/3

84-los-principales-logros-de-la-cienciaargentina.html

UNESCO (1998). Conferencia mundial sobre la educación superior. La educación superior en el siglo XXI. Visión y acción. París. http://www.unesco.org/education/educpro g/wche/declaration_spa.htm

Vessuri, H. (1996). Pertinencia de la educación superior latinoamericana a finales del siglo XX. Revista Nueva Sociedad. Buenos Aires, Argentina 